EXEMPLARIa Classica

Journal of Classical Philology

18, 2014, pp. 23-27

ISSN 1699-3225

\title{
UNA CONGETTURA A PSEUDO-SENOFONTE, COSTITUZIONE DEGLI ATENIESI 2.17*
}

\author{
Luigi BatTEZZATo \\ Università degli Studi del Piemonte Orientale \\ luigi.battezzato@lett.unipmn.it
}

SUMMARY

The manuscript text of [X.] Ath. 2.17 gives impossible syntax and meaning: h̀v

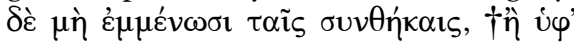

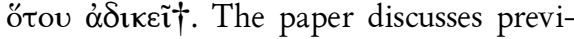
ous emendations, arguing that they are unsatisfactory, and conjectures that the

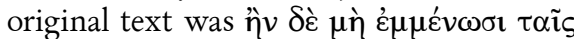

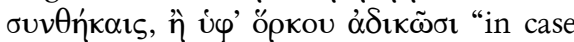
they do not respect the agreements, or violate justice when under oath".

KEYWORDS

Pseudo-Xenophon, Constitution of the Athenians, Old Oligarch, Textual criticism.
RESUMEN

Il testo di [X.] Ath. 2.17, come riportato dai manoscritti, è impossibile per motivi sin-

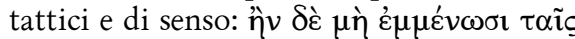

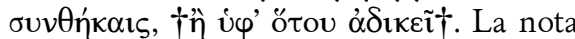
discute proposte precedenti, sostenendo che sono insoddisfacenti, e congettura come il

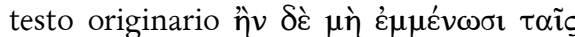

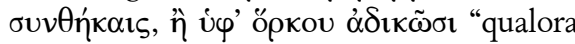
non rispettino i patti, oppure, vincolati da giuramento, commettano ingiustizia”.

Palabras clave

Pseudo-Senofonte, Costituzione degli Ateniesi, Vecchio oligarca, Critica testuale.

Fecha de recepción: 24/01/2014

Fecha de aceptación y versión final: 12/05/2014

L'autore di questo trattato osserva chele democrazie possono più facilmente cambiare alleanze delle oligarchie: sarà sempre possibile ai cittadini dire "io non c'ero" quando si è votata una determinata scelta politica. Invece nei regimi oligarchici i nomi dei capi sono ben noti, e non è possibile nascondersi nell'anonimato della folla. L'autore distorce i fatti: anche nelle democrazie veniva registrato il nome di chi proponeva una deliberazione, e anche le oligarchie prevedevano voti "di massa", ad esempio per acclamazione1.

* Ringrazio E. Medda e i referee anonimi della rivista per suggerimenti e osservazioni.

${ }^{1}$ Cfr. E. C. Marchant, Xenophontis Opera Omnia: V, Opuscula, Oxonii 1920; V. Gray, Xenophon on Government, Cambridge 2007. Per indicazioni sulla manipolazione dei fatti operata dall'anonimo, e su queste pratiche democratiche e oligarchiche si veda J.L. Marr e P.J. 
Questo è il testo dei manoscritti ABCM, stampato da Marchant e da Gray²:

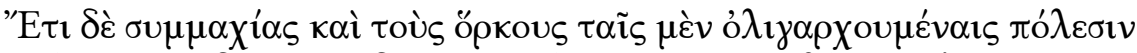

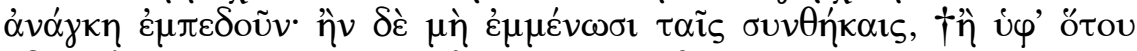

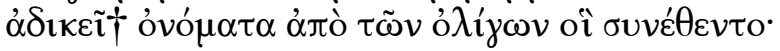

"E inoltre per quanto riguarda le alleanze e i giuramenti, è necessario che le città governate da una oligarchia li rispettino: infatti qualora non rimangano fedeli agli accordi, †oppure sotto la cui spinta uno commetta ingiustizia $\dagger$ i nomi dai pochi che stipularono l'accordo".

Il passo è chiaramente corrotto e/o lacunoso. Le congetture finora proposte non appaiono convincenti per il senso e per probabilità paleografica ${ }^{3}$.

Rhodes, The 'Old Oligarch': The Constitution of the Athenians Attributed to Xenophon, Oxford 2008, 126-8 ad loc.

${ }^{2}$ Anche A. Kirchhoff, Xenophontis qui fertur libellus de republica Atheniensium, editio altera correcta, Berolini 1881 stampa l'incomprensibile testo dei manoscritti ma non segna né crux né congetture in apparato. Si tratta di un testo sintatticamente inaccettabile, come è ovvio dalla traduzione sopra proposta.

${ }^{3}$ Per un ampio elenco di proposte, che nella quasi totalità consistono nell'inserzione di numerosissime parole in lacuna, cfr.l'apparato ad loc. in E. Kalinka, Die Pseudoxenophontische

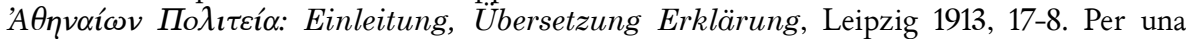

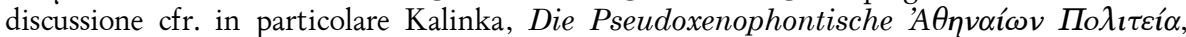
Leipzig-Berlin 1913, 235-6 e W. Lapini, Commento all'Athēnaiōn Politeia dello PseudoSenofonte, Firenze 1997, 223-4 ad loc. La congettura di H. Frisch, The Constitution of the Athenians: A Philological-Historical Analysis of Pseudo-Xenophon's Treatise De Republica Atheniensium, København 1942, 274-5 ad loc. ha goduto di un certo successo: egli

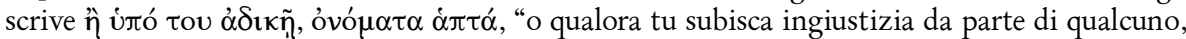

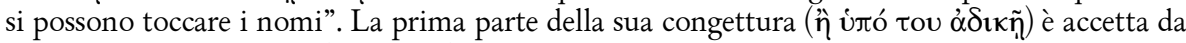
G. W. Bowersock, "Pseudo-Xenophon”, HSPh 71, 1967, 33-55 e G. W. Bowersock, PseudoXenophon, Constitution of the Athenians, new edition with introduction and translation, in G. W. Bowersock-E. C. Marchant, Xenophon: In Seven Volumes. VII, Scripta Minora. London-Cambridge Mass. 1968, L. Canfora, La democrazia come violenza, Palermo 1982, Marr-Rhodes, The 'Old Oligarch', ad loc. Il riferimento a una seconda persona singolare è presente in altre parti del trattato $(1.8 ; 1.10-11)$, ma in riferimento ad un interlocutore che propone obiezioni all'organizzazione democratica di Atene ( $\mathrm{cfr}$. in particolare Marr-Rhodes, The 'Old Oligarch', 169-70). In questa sezione, dedicata ad accordi tra stati, non si vede quale rilevanza abbia rimarcare l'ingiustizia subita da un singolo. Non è nemmeno congruente con il senso del passo che l'ingiustizia sia commessa «da qualcuno»: come si potrebbe scoprire questo singolo individuo nel pur piccolo gruppo di oligarchi? Il gruppo di oligarchi può rifugiarsi dietro la stessa giustificazione dei democratici: "non sono stato io". R. Osborne, The Old Oligarch: Pseudo-Xenophon's Constitution of the Athenians, London 2004, 28 traduce "or if some injustice is committed"; egli segue il testo di Marchant ma dichiara di aver "made some sense of corrupt passages at 2.17" e altrove. Il tuo testo sembra tradurre un adattamento della congettura di Frisch. Per una serrata critica alla congettura di Frisch, si vedano le obiezioni di Lapini, Commento, 223-4 già citato all'inizio della nota. 
Si suggerisce di leggere:

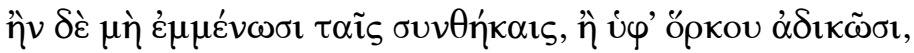

"qualora non rispettino i patti, oppure, vincolati da giuramento, commettano ingiustizia”

Per l'espressione ì $\varphi$ ' ópкou si osservi quello che Edipo dice a Teseo in S. OC 650:

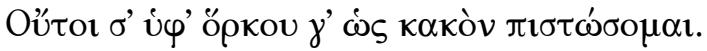

"Non chiederò una garanzia sottoponendoti a un giuramento, come se fossi una persona di cui non fidarsi"

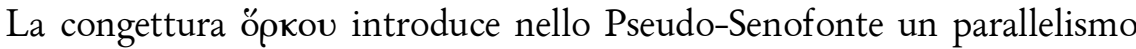
con $\sigma v v \theta \eta \dot{k} \alpha$ ıs. La correlazione tra accordi e giuramenti è attestata da Lys.

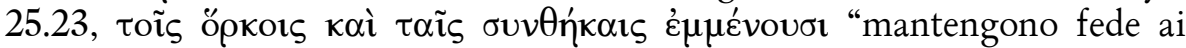
giuramenti e agli accordi", ricordato da Lapini ${ }^{5}$, e da decine di passi negli oratori e in altri scrittori: cfr. ad es. Isoc. $4.81 ; 6.21$ e $27 ; 8.96 ; 14.12,17,23$, $39,44,63$; 18.20, 21, 29, 44, 67; Lys. 2.62; 6.45; 13.88, 89; 25.28, 35; 26.16; D. 15.26; 17.1, 2, 4, 8; 23.10; X. Ages. 1.12. I due termini sono strettamente accoppiati in tutti questi passaggi. E altamente probabile che anche in questo passo del trattato sulla Costituzione degli Ateniesi sotto ötou si celi il termine öркоs.

Su questa linea è naturalmente possibile formulare congetture alternative che si allontanano ulteriormente dal testo dei manoscritti. ${ }^{6}$ In particolare, in congiunzione con un verbo che indica una azione di carattere moralmente

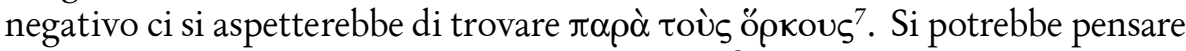

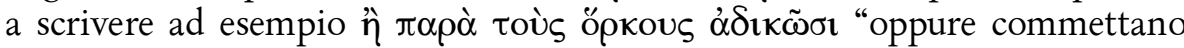

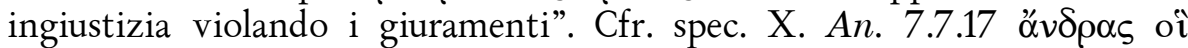

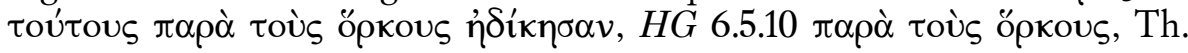

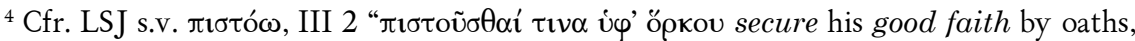

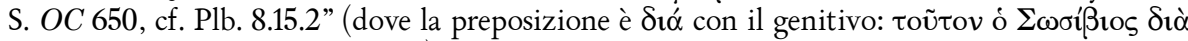

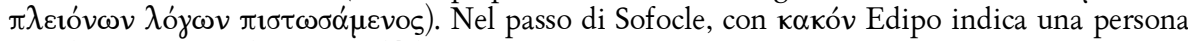
socialmente e moralmente malfidata.

${ }^{5}$ Cfr. Lapini, Commento, 224.

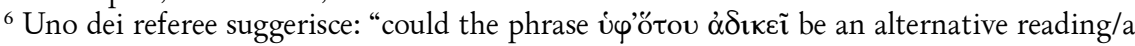
gloss originally introduced by $\grave{\eta}$ in the margin of a ms and inadvertently taken over by a careless scribe in the main text? If so, one would have rather to look for the phrase that this gloss was supposed to replace." In questo caso il testo originario sarebbe difficilmente recuperabile. Una corruzione profonda non può essere esclusa del tutto, anche se questa soluzione è meno economica rispetto a interventi congetturali limitati.

${ }^{7}$ Ringrazio Enrico Medda per aver sollevato questa obiezione. 


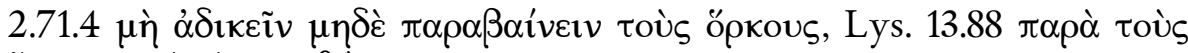

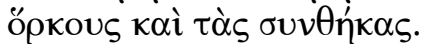

Si trovano però alcuni casi di espressioni in cui un verbo che indica il commettere ingiustizia è strettamente associato a una espressione che indica "sotto giuramento": si veda in particolare Aeschin. 3.99:

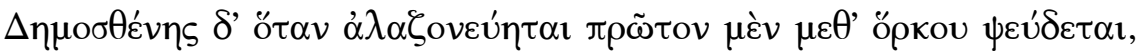

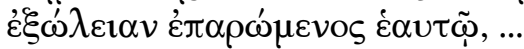

"Ma Demostene, quando vi inganna, prima di tutto mente sotto giuramento, invocando la rovina per se stesso, ..."

e [D.] 52.31

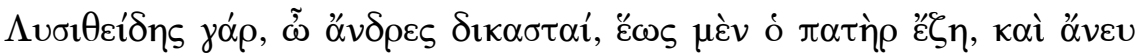

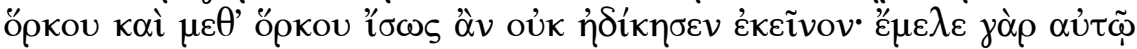

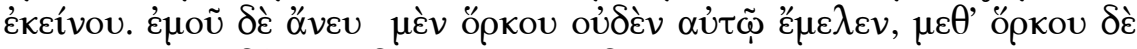

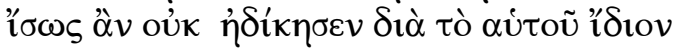

"Lisitide infatti, o uomini della giuria, finché era vivo mio padre forse non avrebbe commesso ingiustizia contro di lui, sia senza giuramento che sotto giuramento; infatti di lui si curava. Di me, senza giuramento, non gli importava nulla; ma contro di me, sotto giuramento, forse non avrebbe commesso ingiustizia a motivo del suo interesse privato".

Naturalmente sono molto frequenti anche frasi in cui un participio concessivo "pur avendo giurato..." è correlato con un verbo sovraordinato che indica la violazione del giuramento8.

La parola "giuramenti" è già usata dall'autore nella frase precedente; lo stile del trattato è molto ripetitivo", e la corruzione si sarà verificata per semplice

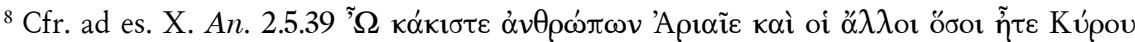

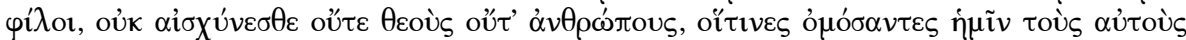

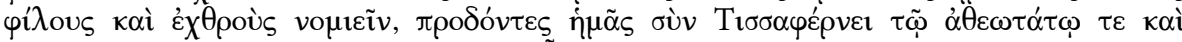

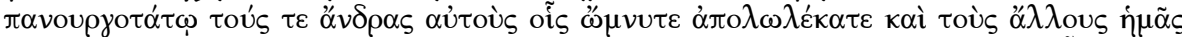

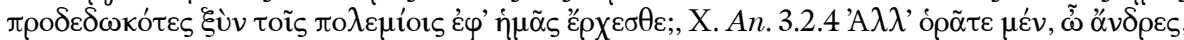

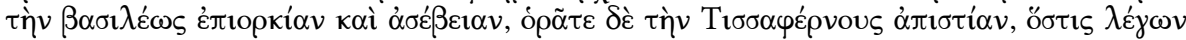

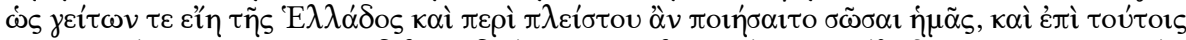

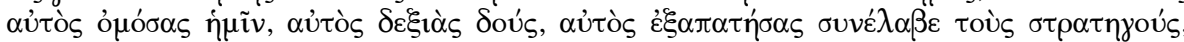

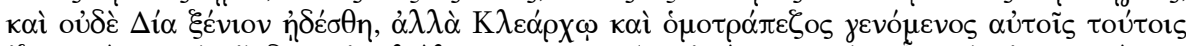

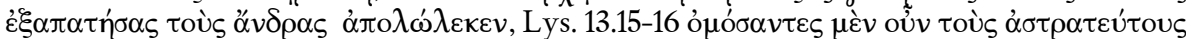

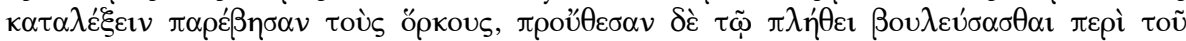

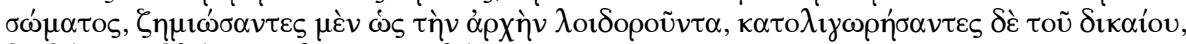

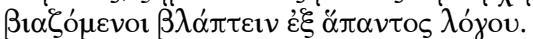

${ }^{9}$ Cfr. M. Joyal, "The language and style of the Old Oligarch", in R.B. Egan, M. Joyal, 
errore grafico o per semplificazione fonetica del gruppo di consonanti. La seconda parte della frase rimane corrotta o lacunosa.

eds., Diamonopylai: Essays in Classics and the Classical Tradition Presented to E.G. Berry, Winnipeg 2004, 221-39, spec. 221-8; Marr-Rhodes, The 'Old Oligarch', 27: "Close verbal repetition is a very marked stylistic feature of X's work [con X Marr e Rhodes designano l'anonimo autore del trattato]. Again and again we find the same word, or cognate forms of that word, used two, three or more times within the space of a few sentences". 
